\title{
Rheology and the Eye: blood, tears and other humours
}

\section{Eye (2018) 32, 163; doi:10.1038/eye.2017.311}

The Cambridge Ophthalmological Symposium was one of the many valued innovations brought to the field of ophthalmology by the late Peter Watson, former editor of this journal, who sadly passed away in 2017. The concept was both novel and unique: a 2-day residential meeting bringing together basic scientists and clinicians to discuss a well defined topic within ophthalmology in detail under the chairmanship of one of the leaders in that field.

Now in its 47th year, the topic of 'Rheology and the Eye' saw basic scientists, chemical engineers, physicists, veterinary surgeons, clinical scientists, ophthalmologists, and cardiologists discuss the rheology and interactions of blood, tears, aqueous, and vitreous, as well as the associated rheology of imaging and surgery as applied to field of ophthalmology.

Rheology and ophthalmology are related yet different fields. The clinical science of ophthalmology is intimately involved in fluid currents of various viscosities and indeed
E Stefánsson ${ }^{1}$ and MP Snead ${ }^{2}$

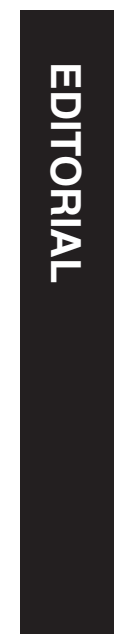

ophthalmological treatment in many cases influences the formation, extraction, currents, and accumulation of various fluids in the eye, including aqueous and vitreous humour, blood, and tears. These fluids are governed by the laws of physics and the relevant field is rheology. By bringing together experts in the basic science of rheology and the clinical science of ophthalmology, we attempt to find the solutions to some of the clinical problems we face in ophthalmology.

This issue of 'Eye' brings together in hard copy record the distillate of each contribution-all from international leaders in their field. By so doing, the aspiration is that novel lines of enquiry and cross-specialty collaborations may emerge from both clinicians and scientists who might otherwise be unaware of the potential collaborative lines of enquiry for the advancement of understanding of our specialty.

\section{Conflict of interest}

The authors declare no conflict of interest.

\author{
${ }^{1}$ University of Iceland, \\ National University \\ Hospital, Reykjavík, Iceland \\ ${ }^{2}$ Vitreoretinal Service, \\ Cambridge University NHS \\ Foundation Trust, \\ Addenbrooke's Hospital, \\ Cambridge, UK \\ Correspondence: \\ MP Snead, Vitreoretinal \\ Service, Cambridge \\ University NHS Foundation \\ Trust, Addenbrooke's \\ Hospital, Hills Road, \\ Cambridge CB20QQ, UK \\ Tel: +440 1223348 842; \\ Fax: +4401223217054. \\ E-mail: mps34@cam.ac.uk
}

